Article

\title{
Research on a Nonlinear Dynamic Incipient Fault Detection Method for Rolling Bearings
}

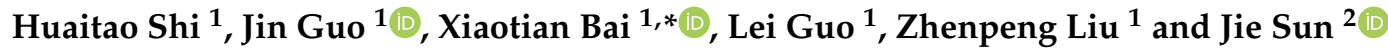 \\ 1 School of Mechanical Engineering, Shenyang Jianzhu University, Shenyang 110168, Liaoning, China; \\ sht@sjzu.edu.cn (H.S.); guojin@stu.sjzu.edu.cn (J.G.); guolei@stu.sjzu.edu.cn (L.G.); lzp@sjzu.edu.cn (Z.L.) \\ 2 The State Key Laboratory of Rolling and Automation, Northeastern University, Shenyang 110819, \\ Liaoning, China; sunjie@ral.neu.edu.cn \\ * Correspondence: baixt@sjzu.edu.cn; Tel.: +86-1552-425-1266
}

Received: 28 February 2020; Accepted: 30 March 2020; Published: 3 April 2020

\begin{abstract}
The incipient fault detection technology of rolling bearings is the key to ensure its normal operation and is of great significance for most industrial processes. However, the vibration signals of rolling bearings are a set of time series with non-linear and timing correlation, and weak incipient fault characteristics of rolling bearings bring about obstructions for the fault detection. This paper proposes a nonlinear dynamic incipient fault detection method for rolling bearings to solve these problems. The kernel function and the moving window algorithm are used to establish a non-linear dynamic model, and the real-time characteristics of the system are obtained. At the same time, the deep decomposition method is used to extract weak fault characteristics under the strong noise, and the incipient failures of rolling bearings are detected. Finally, the validity and feasibility of the scheme are verified by two simulation experiments. Experimental results show that the fault detection rate based on the proposed method is higher than $85 \%$ for incipient fault of rolling bearings, and the detection delay is almost zero. Compared with the detection performance of traditional methods, the proposed nonlinear dynamic incipient fault detection method is of better accuracy and applicability.
\end{abstract}

Keywords: rolling bearings; incipient fault detection; feature extraction; timing correlation; nonlinear characteristics

\section{Introduction}

The rolling bearing is one of the most commonly used components in rotating machinery and equipment $[1,2]$. However, the running state of the rolling bearings are easily affected by shock and vibrations, and incomplete statistics show that about $30 \%$ of rotating machinery failures are caused by bearing failures [3,4]. The failures of rolling bearings often develop from the normal stage to the incipient stage, then enter the stage of repeated failure, and finally reach the stage of complete breakdown. The serious faults do not occur in an instant, but has a process of gradual deterioration [5-7]. Therefore, it is of great significance to study the incipient fault detection of rolling bearings, find the incipient signal characteristics of faults, and eliminate the safety hazards in time when the fault has not developed toward a serious degree [8,9].

The fault detection methods mainly include model-driven and data-driven methods. Some scholars have established rolling bearings degradation models by analyzing the prior physical knowledge of rolling bearings $[10,11]$, or completed the incipient fault detection of rolling bearings by analyzing the characteristic frequency of failure of rolling bearings [12,13]. However, the model-based method requires an accurate prior physical model of rolling bearings. The motion and internal stress of rolling bearings are complicated, which causes the decline of accuracy and generalization. The data-driven method is used to finding incipient failures by analyzing the collected data, and is more suitable 
for incipient failure detection of current large-scale industrial processes. The deep learning theory brings opportunities to solve the feature extraction problems in rolling bearings fault detection with its powerful feature extraction capabilities [14-17]. By continuously increasing the abstraction of various representations, rich and differentiated features can be obtained and studied. However, the computational intensity of the complicated model becomes stronger, which will lead to a decrease in the training and detecting speed of the system in a large network structure. The method based on signal processing is also a common detection method for incipient faults [18-20]. For example, the signal feature processing based on the wavelet variation method [21] and empirical mode decomposition method [22] has been generalized successfully in many applications. However, the incipient fault features are usually weak and buried in the noises, and the noise in the entire signal makes the normal state similar to the actual incipient fault, thus affecting the effect of fault detection.

Many scholars have proposed many methods for fault detection, but incipient fault detection still mainly focuses on the following challenges [23-25]: 1. The incipient failure characteristics are weak, and it is difficult to extract the fault characteristics. 2. The vibration signal is a kind of non-linear and non-stationary signal, which brings difficulty to extract the rolling bear fault weak feature 3 . The collected signals can be easily affected by the noise and transmission loss, which makes it difficult to extract signal characteristics. Therefore, the weak fault feature extraction is the key technology for incipient fault detection of rolling bearings.

Literature [26] proposed an incipient fault detection method based on the Deep Principal Component Analysis (Deep PCA). This method is used to the principle of deep decomposition to accurately divide the data to extract weak fault features and detect incipient faults. However, Deep PCA is a linear stationary transformation method, when it is used for a monitoring non-linear time-varying system, its performance is greatly reduced since it does not fully take account of the characteristics of the actual system. In order to solve this problem, many non-linear time-varying fault detection methods have been proposed [27-30]. Schölkopf found that the Kernel Principal Component Analysis (KPCA) was a common nonlinear principal component analysis method when researching support vector classification algorithms [31]. It maps the input vector to a high-dimensional space through some preselected non-linear mapping, so that the input vector has better separability, and then performs linear principal component analysis on the mapped data in the high-dimensional space to obtain the non-linear principal components. However, the vibration signals are time series data arranged according to the time axis, and the system model will change gradually when the bearing is running. Normal parameters often deviate from the statistical boundary of the model, causing the original model to lose its existing effect and lead to a false alarm.

Aiming at the problems brought by the weak incipient fault characteristics, this paper proposes a nonlinear dynamic incipient fault detection method for rolling bearings. This method first establishes a dynamic kernel matrix by Dynamic Kernel Principal Component Analysis (DKPCA) method. Then the moving window algorithm is used to update the latest samples to the dynamic kernel matrix and discard the oldest samples without losing the time dynamics of the data during the model building process. Finally, the updated matrix is decomposed into multiple subspaces for detection, and fault information is fully mined. Based on this, the model update process is improved through the autocorrelation matrix, which simplifies the recursive formula and improves the calculation speed.

The rest of the paper is arranged as follows. The basic theory is introduced in the second part. The nonlinear dynamic incipient fault detection method is proposed in the third part. The feasibility of the proposed method is proved by simulation experiments in the fourth part. The conclusion of proposed method is made in the fifth part. 


\section{Preliminary}

\subsection{Kernel Principal Component Analysis Method}

Kernel Principal Component Analysis (KPCA) is a kind of non-linear principal component analysis [32]. This method uses kernel functions to map non-linear data to high-dimensional linear space, and performs linear principal component analysis on the mapped data in high-dimensional space. KPCA can effectively eliminate redundancy among data and spatial correlation, non-linear feature principal components for extracting main data information.

Let the data set $=\left[x_{1}, x_{2} \ldots, x_{n}\right]^{T} \in R^{n * m}$, where $n$ is the sample number and $\mathrm{m}$ is the observed variable. Formula (1) is the kernel function $K\left(x_{i}, x_{j}\right)$ :

$$
K\left(x_{i}, x_{j}\right)=\exp \left(-\frac{\left\|x_{i}-x_{j}\right\|^{2}}{2 \sigma^{2}}\right)
$$

The flow chart of KPCA is shown in Figure 1:

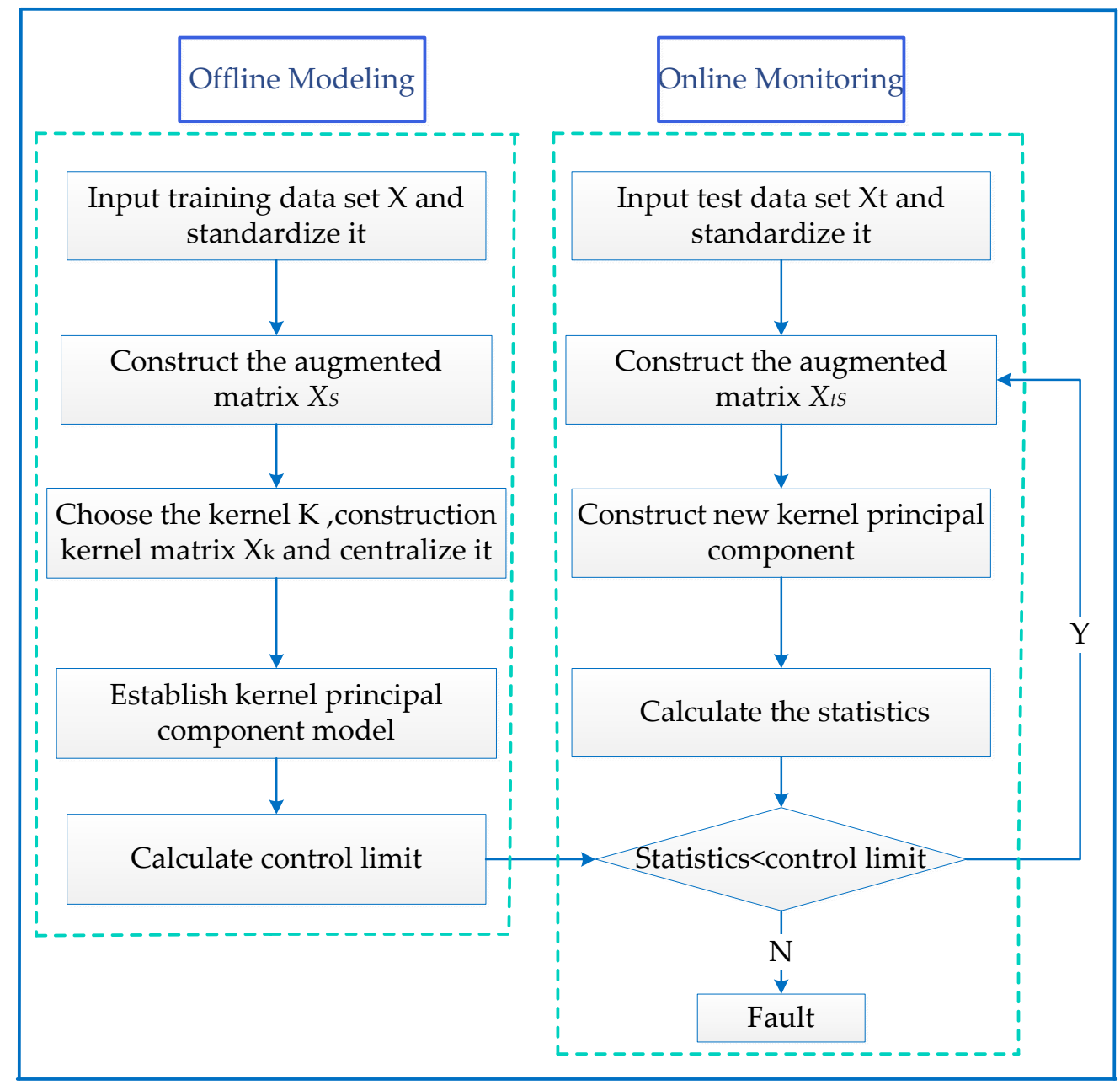

Figure 1. The flow chart of Kernel Principal Component Analysis (KPCA).

\subsection{Adaptive Dynamic Method}

The vibration signal collected during the running of rolling bearings reflects the change of the bearing with the running time [33,34]. Aiming at this time correlation, a moving window algorithm was used to update the model in real time to achieve adaptive detection. On this basis, combined with the autocorrelation matrix, the online update speed was approximately doubled. 
In the process of the update model, the recursive moving window is 1 , and the update window length is $L$, that is, the covariance matrix is updated every $L$ data collection. Let the mean value $\bar{X}_{a}$ and the covariance $R_{a}$ of the previous matrix block $X_{a}$ be known then the mean value $\bar{X}_{m}$ and covariance $R_{m}$ of $X_{m}$ is:

$$
\begin{gathered}
\bar{X}_{m}=\frac{L}{L-1} \bar{X}_{a}-\frac{1}{L-1} x_{a} \\
R_{m}=\frac{L-1}{L-2}\left(R_{a}-\frac{1}{L} x_{a-L-1} x_{a-L-1}^{T}-\sum_{a}^{-1} \Delta \bar{X}_{m a} \Delta \bar{X}_{m a}^{T} \sum_{a}^{-1}\right)
\end{gathered}
$$

According to $\bar{X}_{m}$ and $R_{m}$, the mean value $\bar{X}_{a+1}$ and covariance $R_{a}$ of $X_{a}$ as follows:

$$
\begin{gathered}
\bar{X}_{a+1}=\frac{L-1}{L} \bar{X}_{m}+\frac{1}{L} x_{a+L} \\
R_{a+1}=\frac{L-2}{L-1} R_{m}+\frac{1}{L-1} x_{a+L} x_{a+L}^{T}+\sum_{a+L}^{-1} \Delta \bar{X}_{m(a+L)} \Delta \bar{X}_{m(a+L)}^{T} \sum_{a+L}^{-1}
\end{gathered}
$$

where, $\Delta \bar{X}_{m a}=\bar{X}_{m}-\bar{X}_{a}, \Delta \bar{X}_{m(a+1)}=\bar{X}_{a+1}-\bar{X}_{m}$.

According to Formulas (2)-(5), updating the covariance can avoid the complexity of calculating large block matrices and matrix multiplication, but the calculation steps are still complicated. The online update rate can quickly increased through the autocorrelation matrix:

$$
\begin{gathered}
\sum_{a}^{-1} \Delta \bar{X}_{m a} \Delta \bar{X}_{m a}^{T} \sum_{a}^{-1}=\frac{1}{L^{2}} x_{a} x_{a}^{T} \\
\sum_{a+L}^{-1} \Delta \bar{X}_{m(a+L)} \Delta \bar{X}_{m(a+L)}^{T} \sum_{a+L}^{-1}=\frac{1}{L^{2}} x_{a+L} x_{a+L}^{T}
\end{gathered}
$$

Substitute Formulas (3), (6), and (7) into Formula (5) obtaining the Formula (8):

$$
R_{a+1}=R_{a}+\frac{L^{2}+L-1}{L^{2}(L-1)}\left(x_{a+L} x_{a+L}^{T}-x_{a} x_{a}^{T}\right)
$$

The improved model only needs to directly calculate $X_{a+1}$ through $X_{a}$, which reduces the calculation of the number of steps from four to just two, and the online update rate is improved by double. It saves the storage space of hardware and greatly improves the calculation efficiency.

\section{Nonlinear Dynamic Incipient Fault Detection Method}

Due to the KPCA dynamic model method discards some fault features when extracting the main components, which lead to this method cannot accurately detect weak fault information. This paper combining PCA a deep decomposition theory proposes a nonlinear dynamic incipient fault detection method based on Deep Adaptive Dynamic Kernel Principal Component Analysis (Deep ADKPCA). This method decomposes the data set and retains most of the fault information, which can fully mine the data to obtain more accurate fault information to achieve the purpose of incipient fault detection.

\subsection{The Deep Decomposition Principle}

In order to even better extract the feature of the weak fault in the vibration signal, combining the deep decomposition theorem, the original space is decomposed into multiple data processing layers, and the weak fault information in the signal is deeply mined to achieve the effect of incipient fault detection.

According to the method in Section 2.1, the dynamic kernel matrix was decomposed into the principal component space and the residual space is shown in the Formula (9):

$$
X_{K}=X_{K 11}+X_{K 12}
$$


where, $X_{K 11}$ and $X_{K 12}$ represent the first-order subprincipal component space and subresidual space of the original dynamic kernel matrix $X_{K}$, respectively. It can be expressed as Formula (10):

$$
\begin{gathered}
X_{K 11}=P_{11} P_{11}^{T} X_{K} \\
X_{K 12}=\left(I-P_{11} P_{11}^{T}\right) X_{K}
\end{gathered}
$$

where, $P_{11}$ is the principal feature vector of $X_{K}$. Continue to divide $X_{K 11}$ and $X_{K 12}$ to obtain the second-order subprincipal component space $X_{K 21}, X_{K 23}$ and subresidual space $X_{K 22}, X_{K 24}$. By analogy, the original dynamic kernel matrix $X_{K}$ can be expressed as the sum of $2^{i}$ subspaces, where $i$ represents the order. The subspace $X_{K i j}$ can be expressed as Formula (11):

$$
X_{K i j}=\left\{\begin{array}{l}
P_{i j} P_{i j}^{T} X_{K i-1,(j+1) / 2} j \text { is odd } \\
\left(I-P_{i j} P_{i j}{ }^{T}\right) X_{K i-1, \frac{j}{2}} j \text { is even }
\end{array}\right.
$$

By increasing the order of the Deep ADKPCA method, extremely weak fault information can be detected, thereby achieving incipient fault detection. The structure of Deep ADKPCA is shown in Figure 2:

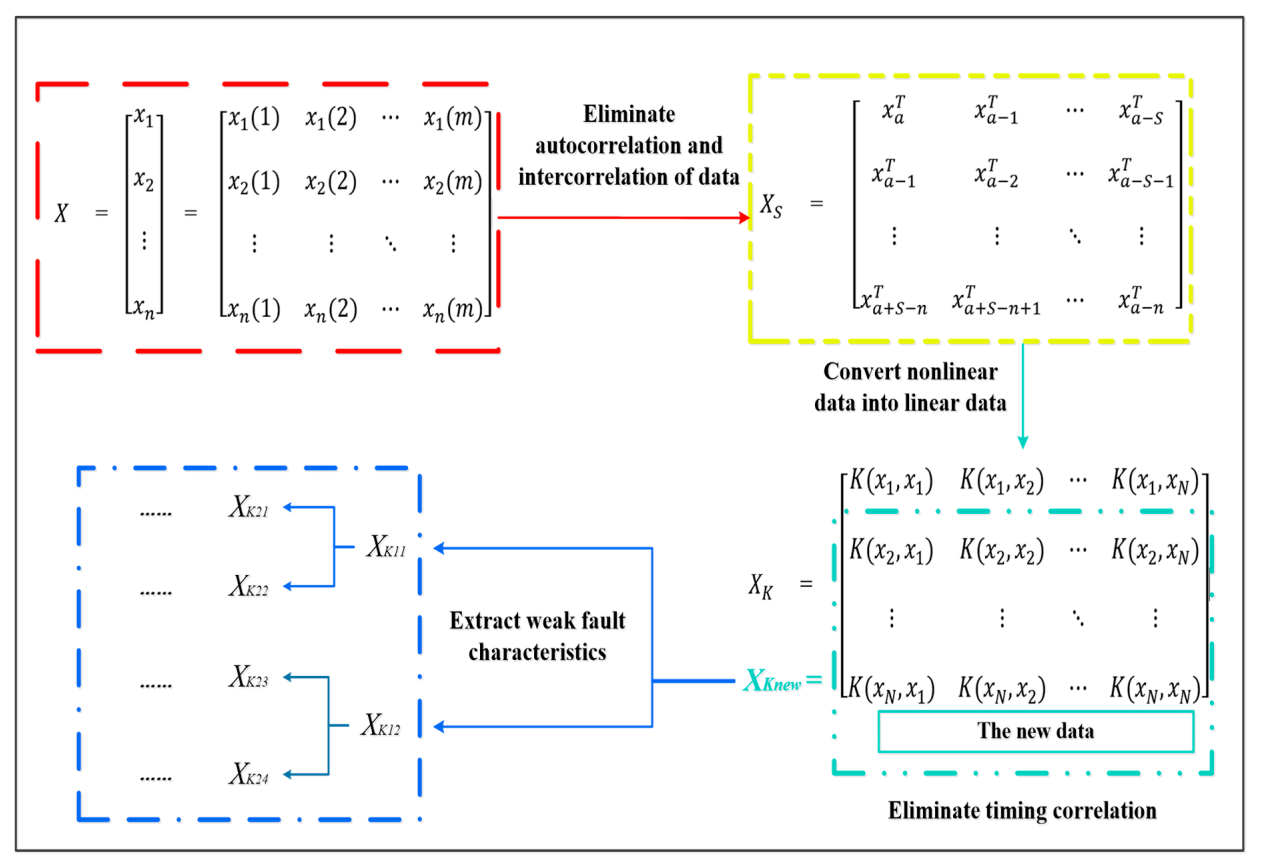

Figure 2. The structure of Deep Adaptive Dynamic Kernel Principal Component Analysis (Deep ADKPCA).

\subsection{The process of Deep ADKPCA}

Firstly, the EMD method was used to decompose the vibration signal to obtain $n$ IMF components, secondly the $n$ IMF components were composed into a matrix, and thirdly the matrix was obtained by kernel transformation to obtain a dynamic kernel matrix. Eigenvalue decomposition was performed on the dynamic kernel matrix to obtain the principal component space and residual space. Finally, the subprincipal component space and the subresidual space were decomposed multiple times to obtain $i$-layer subspaces, calculate the statistics, and control limits of each subspace and compare them to determine whether a fault occurs.

The $T_{i j}^{2}$ and $S P E_{i j}$ statistics for each subspace $X_{K i j}$ as Formulas (12) and (13):

$$
T_{i j}^{2}=X_{K}^{T} P_{i+1,2 j-1} \Lambda_{i j}^{-1} P_{i+12 j-1}^{T} X_{K}
$$




$$
S P E_{i j}=\sum_{c=1}^{n} t_{i j_{c}}^{2}-\sum_{c=1}^{a_{i j}} t_{i j_{c}}^{2}
$$

where, $a_{i j}$ is the number of principal elements of the subspace $X_{K i j}$.

The $T_{i j}^{2}$ and $S P E_{i j}$ control the limit for each subspace $X_{K i j}$ :

$T_{i j}^{2}$ satisfies the F distribution, and its control limit is shown in Formula (14):

$$
T_{i j}^{2} \sim \frac{a_{i j}\left(N^{2}-1\right)}{N\left(N-a_{i j}\right)} F_{a_{i j}, N-a_{i j}}
$$

$S P E_{i j}$ satisfies the $\chi^{2}$ distribution, and its control limit is shown in Formula (15):

$$
\delta_{i j}^{2}=g_{i j} \chi^{2}\left(h_{i j}\right)
$$

where, $g_{i j}=\frac{\rho_{i j}^{2}}{2 \mu_{i j}}, h_{i j}=\frac{2 \mu_{i j}^{2}}{\rho_{i j}^{2}} . \mu_{i j}$ and $\rho_{i j}^{2}$ are the mean and variance of $S P E_{i j}$.

The flowchart of the fault detection method based on Deep ADKPCA is shown in Figure 3:

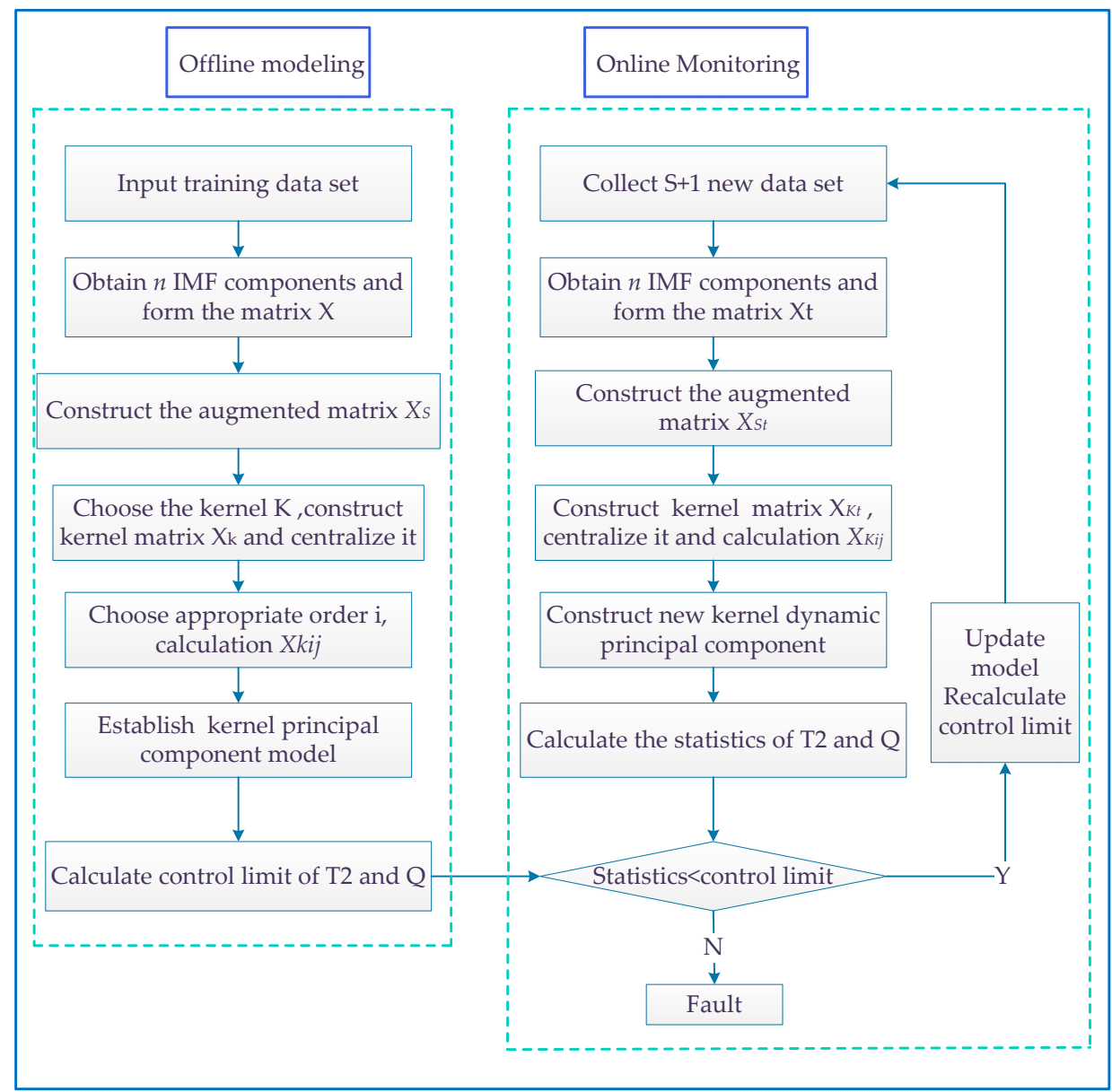

Figure 3. The process of Deep Adaptive Dynamic Kernel Principal Component Analysis (Deep ADKPCA).

\section{Experimental Results and Analysis}

This section used the comprehensive simulation test bench for mechanical failure to perform experiments as shown in Figure 4. The experimental device included a comprehensive mechanical 
fault simulation test rig, AIC9916FS comprehensive fault diagnosis and analysis system software, FL6816L16 channel sensor, BSZ800D-16 vibration signal acquisition instrument, and computer storage device. In the experiment, the speed was $1730 \mathrm{r} / \mathrm{min}$.

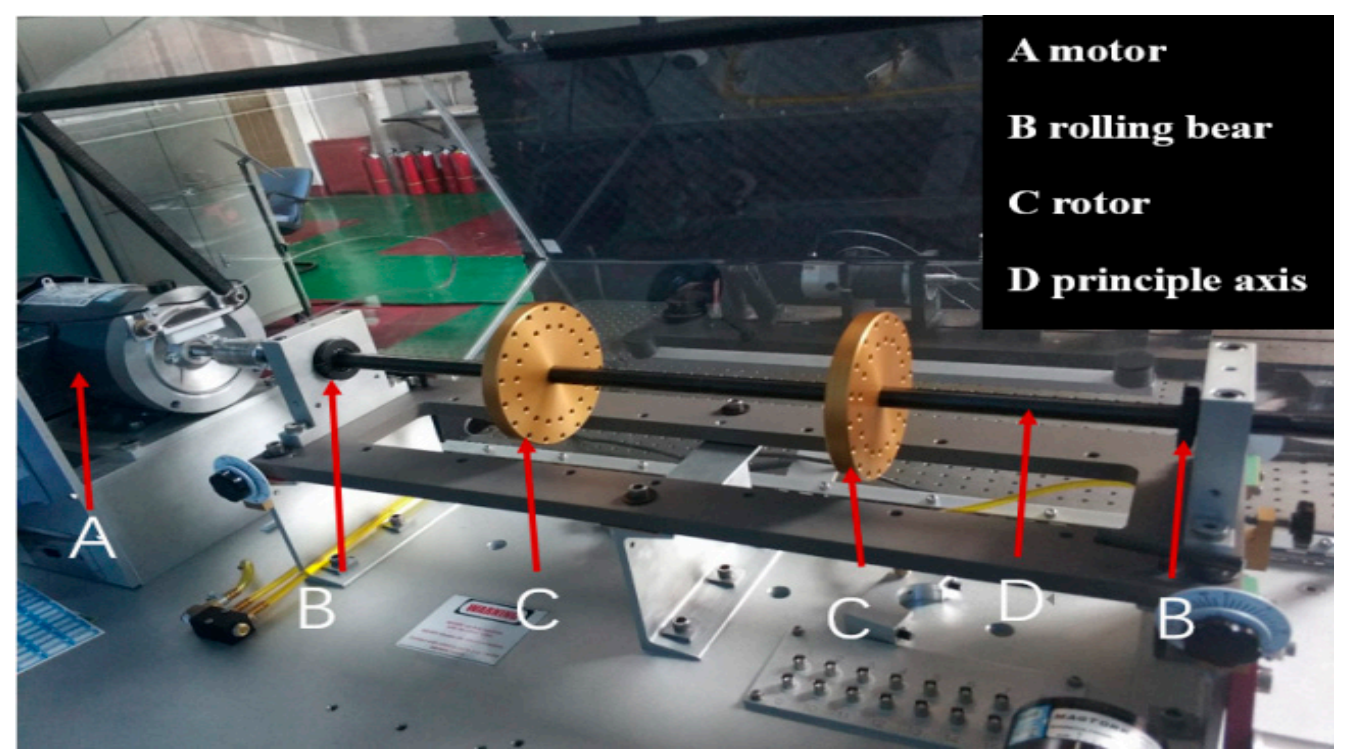

Figure 4. Comprehensive mechanical failure test bench.

There were three sets of data in the experiment. The first set was the vibration signal of a normal undamaged rolling bearing to establish an offline model. The second set was the vibration signal of the outer ring fault rolling bearing with a fault diameter of $0.2 \mathrm{~mm}$, and the last set was the vibration signal of the rolling body fault rolling bearing with a fault diameter of $0.2 \mathrm{~mm}$. The online monitoring part used the fault data collected by the second and third sets. Each set of vibration signal data collected 1024 samples, that is $n=1024$. The update window size $L=3$. The first control limit was calculated through normal data in the offline modeling process, and then in the online monitoring process, the model was updated every three samples collected. The selected fault diameter was relatively small, and the fault features in the vibration signal were weak, so these faults were accounted as the incipient fault.

\subsection{Simulation Results and Analysis of The Rolling Bearing Outer Ring Faults}

\subsubsection{The Rolling Bearing Outer Ring Fault Simulation Results}

When there is a defect point on the outer surface of a rolling bearing, the rolling bearing passes the defect point during operation, which will generate a periodic pulse force to the system. Generally, because the outer ring is fixed, the pulse intensity of each cycle is considered as constant, and the periodic pulse signal presents the law of periodic equal amplitude vibration changes [35]. This section simulates the incipient failure of the rolling bearing outer ring.

The validity and accuracy of proposed methods were tested, and the results were also compared with other known methods such as ADKPCA, Deep KPCA, and Deep DKPCA.

The original data was divided into multiple subspaces according to the steps in Section 3.2, and calculate the $T_{i j}^{2}$ and $Q_{i j}$ control limits and statistics of each subspace. The results are shown in Figure 5. Figure 5a,b show the simulation results of $T_{01}^{2}$ and $Q_{01}$ statistics of the original space $X$ based on the Deep ADKPCA method, that is, the simulation results based on the ADKPCA method. Figure $5 \mathrm{c}-\mathrm{j}$ show the simulation results of $T_{i j}^{2}$ and $Q_{i j}$ statistics of the second-order subspace $X_{21}-X_{24}$ based on the Deep ADKPCA method, respectively. In the figures, the solid black line is the statistic, and the red dotted line is the control limit with $95 \%$ confidence. If the statistics exceeded the control 
limit, a fault occurred, otherwise it is normal. Since the model was updated every three sets of data when updating the model, the number of samples in the figure was about 340 . From Figure $5 a, b, T_{01}^{2}$ and $Q_{01}$ were slightly affected by this fault. Figure $5 \mathrm{~d}$,f shows the detection results of incipient failures of rolling bearings outer rings of $Q_{21}$ and $Q_{22}$, we could obtain that $Q_{21}$ and $Q_{22}$ were not sensitive to this fault. Figure $5 \mathrm{~g}$, i show the results of the $T_{23}^{2}$ and $T_{24}^{2}$ statistics on the fault detection. From the image, it can be seen that $T_{23}^{2}$ and $T_{24}^{2}$ were more sensitive to the fault, the fault detection rate was around $90 \%$. So, Deep ADKPCA the method had a good incipient fault detection capability.

Figure 6a-h describes the simulation results of $T_{i j}^{2}$ and $Q_{i j}$ statistics of the second-order subspace $X_{21}-X_{24}$ based on the Deep KPCA method, respectively. Figure $6 \mathrm{~b}, \mathrm{c}, \mathrm{d}$ shows the detection results of incipient failures of rolling bearing outer rings of $T_{22}^{2}, Q_{21}$, and $Q_{22}$, we could obtain that $T_{22}^{2}, Q_{21}$, and $Q_{22}$ were less affected by this fault. From Figure $6 \mathrm{~g}$, we could obtain that $T_{24}^{2}$ was more sensitive to the fault. The Deep KPCA method has some incipient fault detection capabilities, but it still has some disadvantages compared to the Deep ADKPCA method.

Figure 7a-h describes the simulation results of $T_{i j}^{2}$ and $Q_{i j}$ statistics of the second-order subspace $X_{21}-X_{24}$ based on the Deep DKPCA method, respectively. Figure 7a,g describes the detection results of incipient failures of rolling bearing outer rings of $T_{21}^{2}$ and $T_{24}^{2}$, the rolling bearing outer rings fault could be successfully detected by $T_{21}^{2}$ and $T_{24}^{2}$. From Figure $7 \mathrm{c}$, we could obtain that this fault had no obvious influence on $T_{22}^{2}$. 


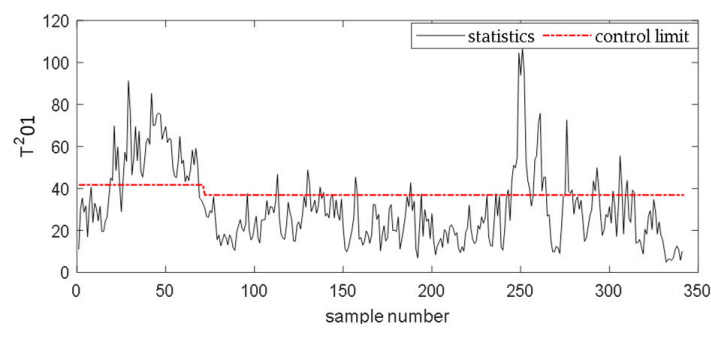

(a)

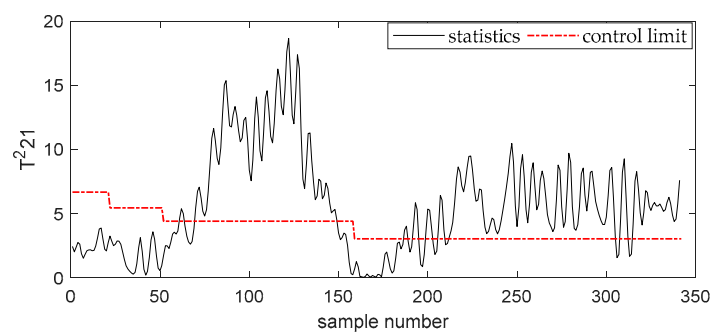

(c)

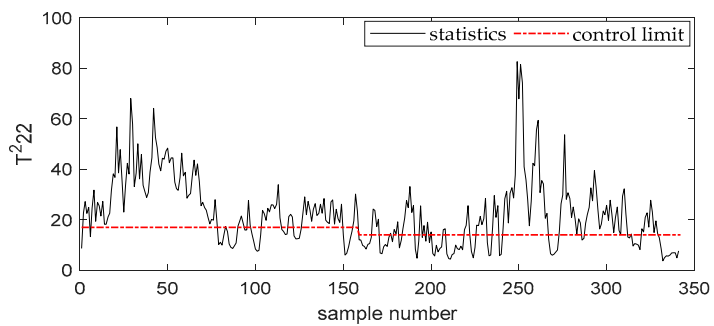

(e)

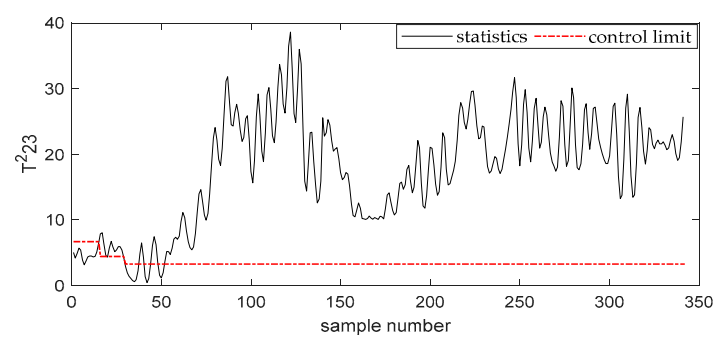

(g)

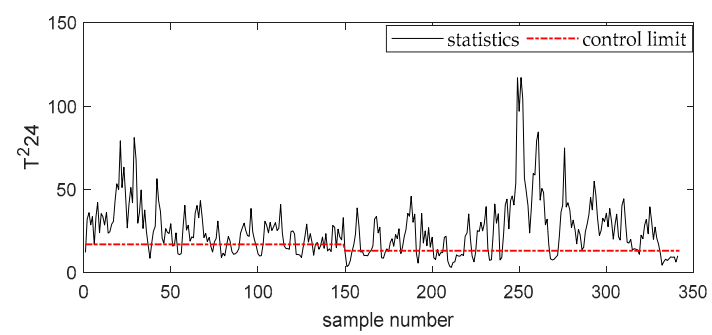

(i)

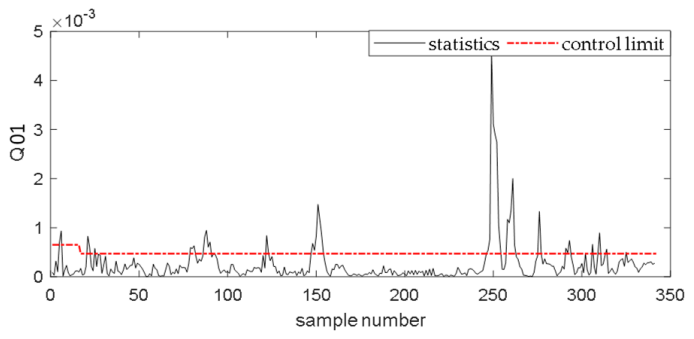

(b)

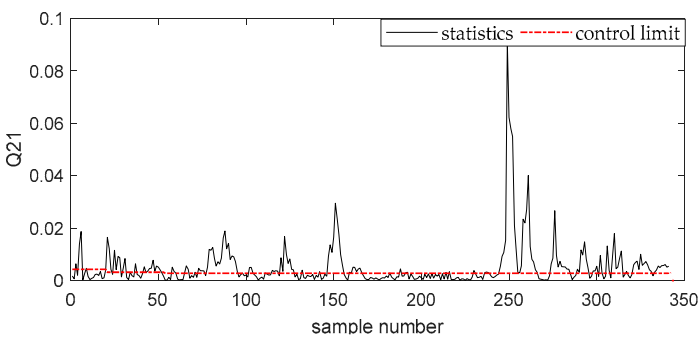

(d)

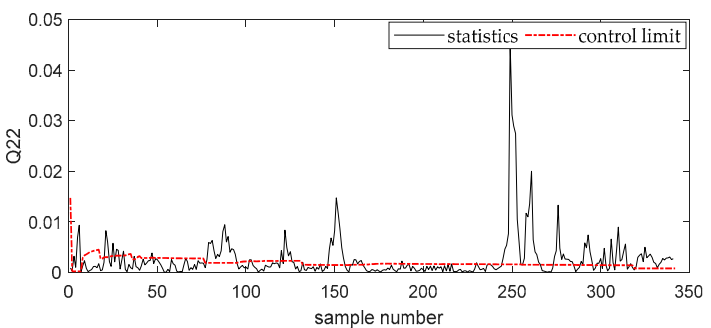

(f)

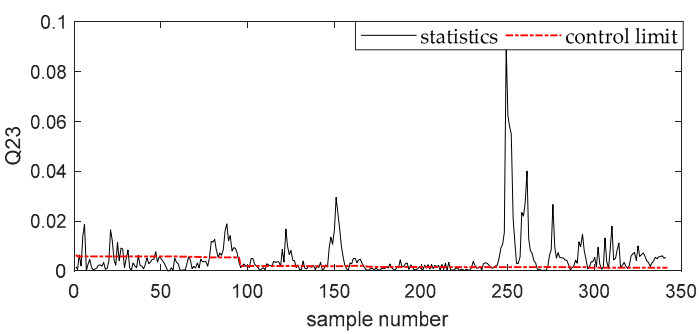

(h)

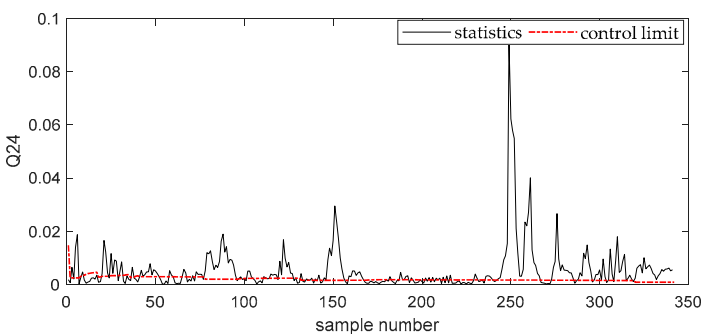

(j)

Figure 5. Experimental results of Deep ADKPCA fault detection method for the rolling bearing outer ring fault. (a) The result of $T_{01}^{2}$, (b)The result of $Q_{01}$, (c) The result of $T_{21}^{2}$, (d) The result of $Q_{21}$, (e) The result of $T_{22^{\prime}}^{2}$ (f) The result of $Q_{22}$, (g) The result of $T_{23^{\prime}}^{2},(\mathbf{h})$ The result of $Q_{23}$, (i) The result of $T_{24}^{2}$ and (j) The result of $Q_{24}$. 


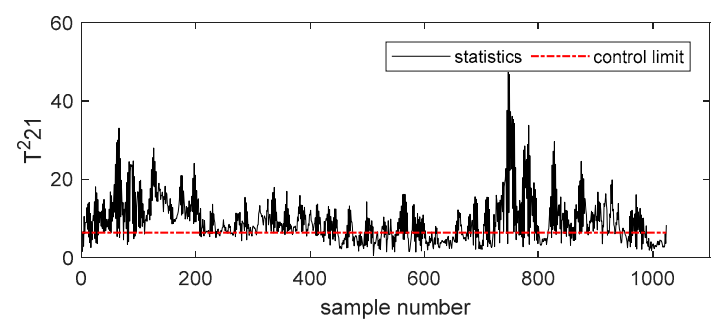

(a)

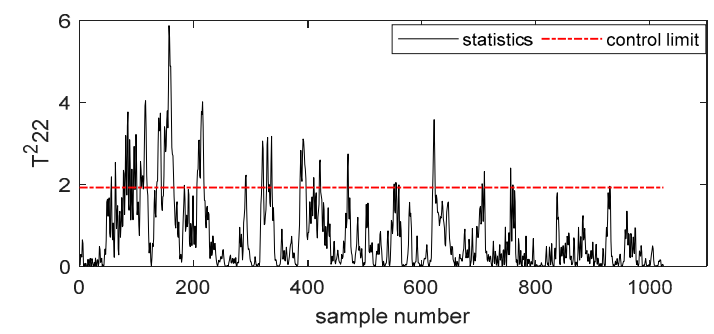

(c)

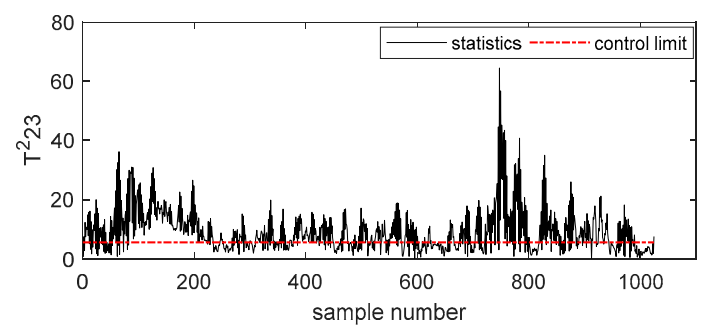

(e)

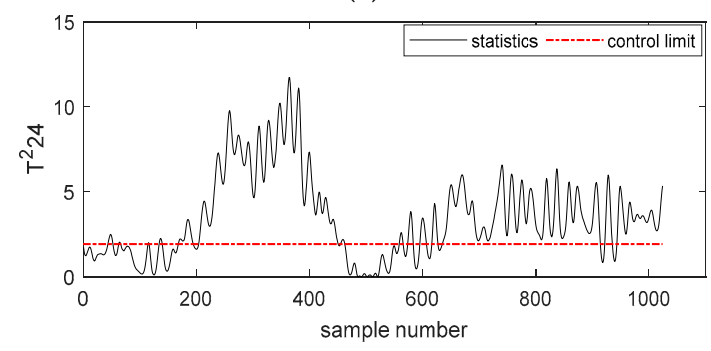

(g)

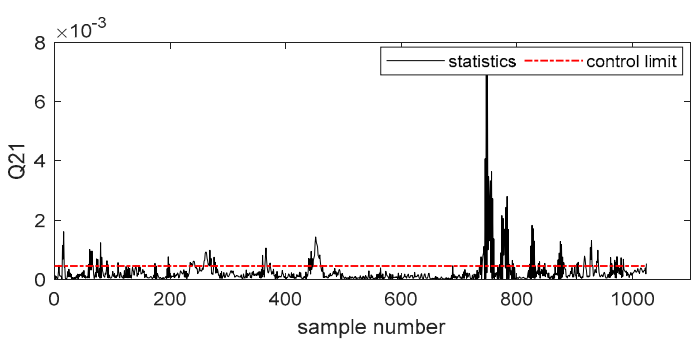

(b)

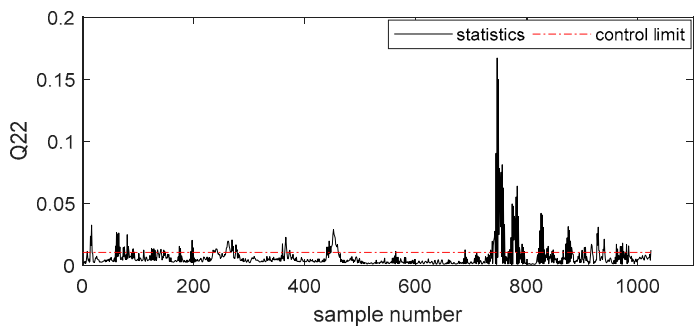

(d)

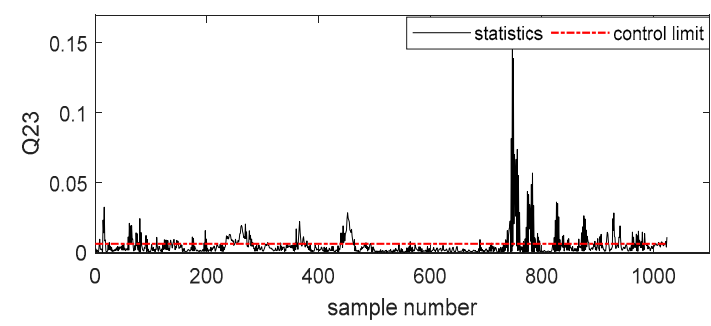

(f)

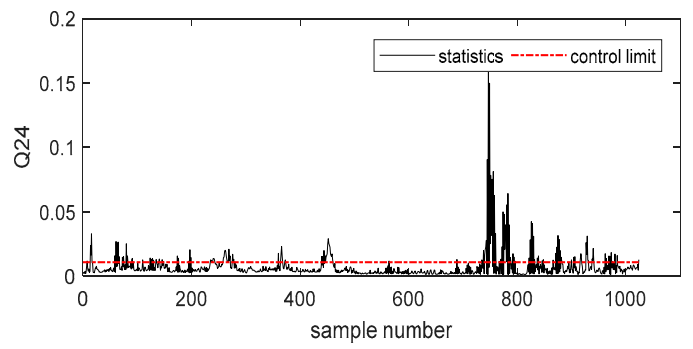

(h)

Figure 6. Experimental results of Deep KPCA fault detection method for the rolling bearing outer ring fault. (a) The result of $T_{21^{\prime}}^{2}$ (b) The result of $Q_{21}$, (c) The result of $T_{22^{\prime}}^{2}(\mathbf{d})$ The result of $Q_{22}$, (e) The result of $T_{23^{\prime}}^{2}$ (b) The result of $Q_{23}$, (g) The result of $T_{24}^{2}$ and (h) The result of $Q_{24}$. 


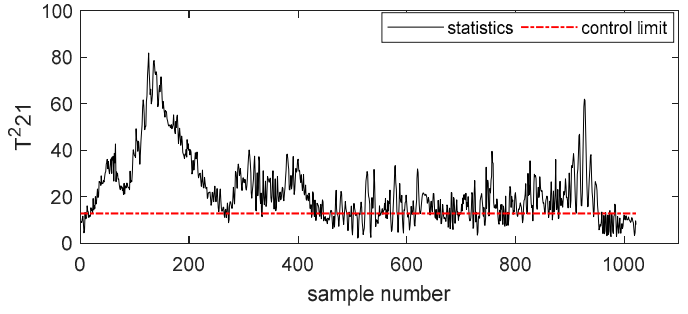

(a)

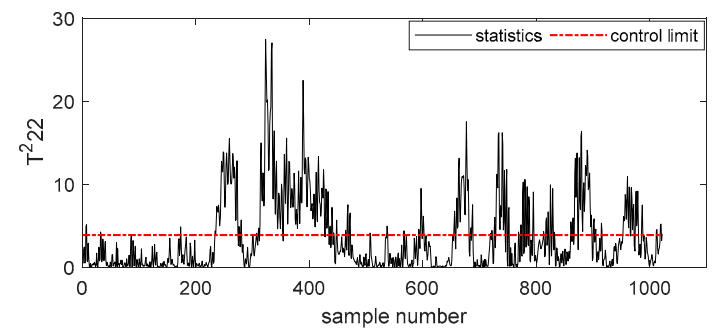

(c)

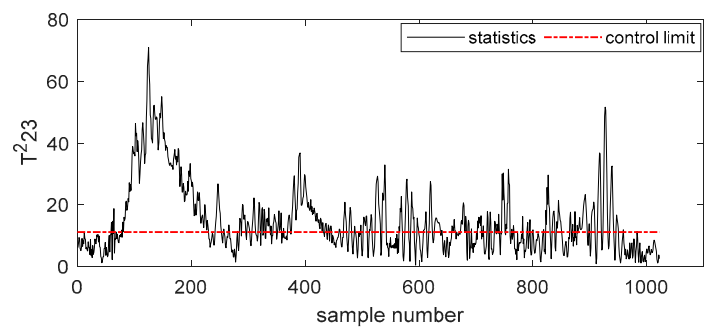

(e)

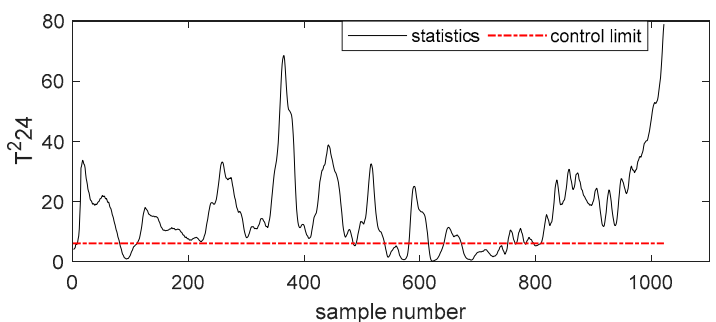

(g)

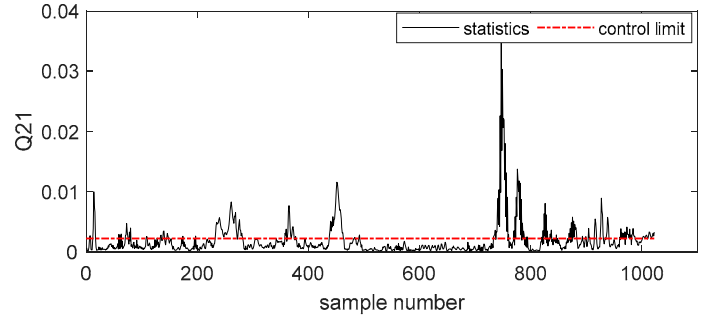

(b)

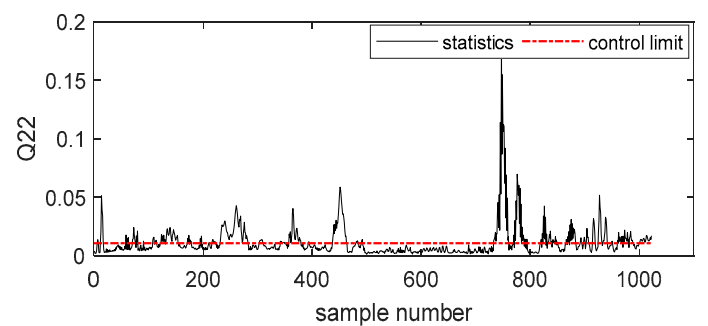

(d)

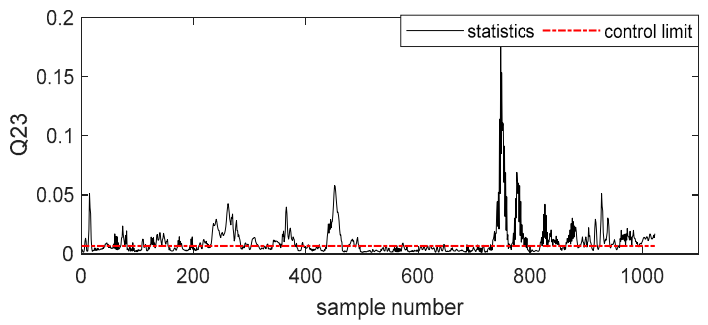

(f)

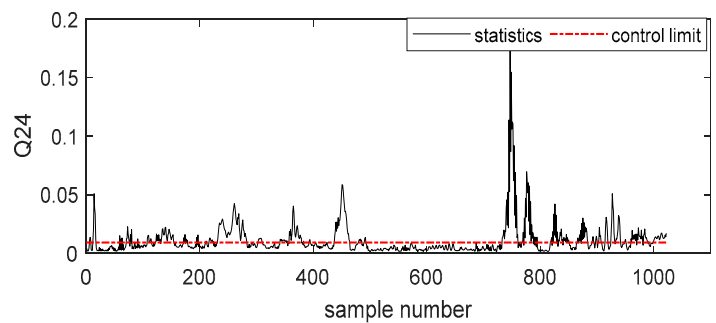

(h)

Figure 7. Experimental results of the Deep DKPCA fault detection method for the rolling bearing outer ring fault. (a) The result of $T_{21}^{2}$, (b) The result of $Q_{21}$, (c) The result of $T_{22^{\prime}}^{2}(\mathbf{d})$ The result of $Q_{22}$, (e) The result of $T_{23^{\prime}}^{2}$ (f) The result of $Q_{23}$, (g) The result of $T_{24}^{2}$ and (h) The result of $Q_{24}$.

\subsubsection{Performance Comparison}

This section compared the experimental results of ADKPCA, Deep DPCA, Deep DKPCA, and Deep ADKPCA by analyzing the fault detection rate and detection delay of the incipient failure experiment results of the rolling bearing outer ring. The detection delay refers to the interval from failure to detection. The fault detection rate refers to the ratio of the number of detected failures to the total number of failures. In the experiments, the thresholds of ADKPCA, Deep DPCA, Deep DKPCA, and Deep ADKPCA method were determined by the same confidence level, and the false detection rate of all methods was similar, so it was not used as the evaluation index.

Figure 8a shows the fault detection rate of rolling bearing outer ring fault by ADKPCA, Deep KPCA, Deep DKPCA, and Deep ADKPCA method. The fault detection rate of the ADKPCA fault detection method was $62.86 \%$. The detection effect by ADKPCA was low, this method basically did not have incipient fault detection capability. The fault detection rate of Deep KPCA and Deep DKPCA was about $80 \%$, compared with ADKPCA method the detection rate had increased, but the detection 
capability still needs to be improved. The fault detection rate of Deep ADKPCA was $92.43 \%$, which was significantly higher than other methods, and had a certain incipient fault detection capability.

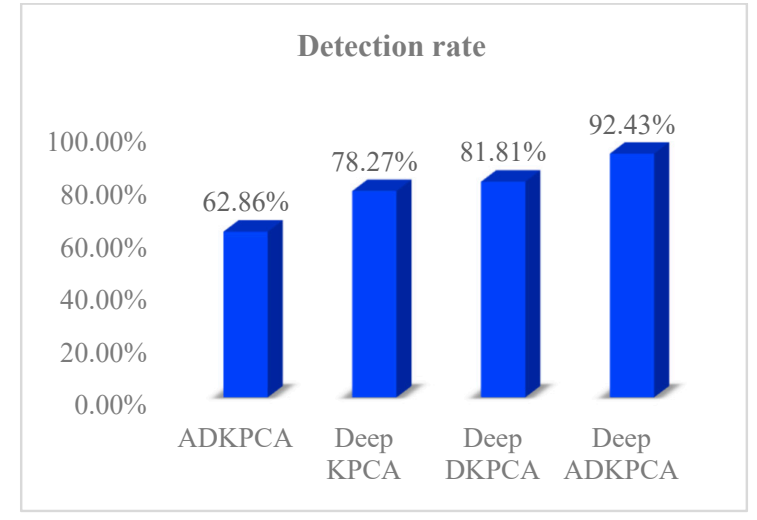

(a)

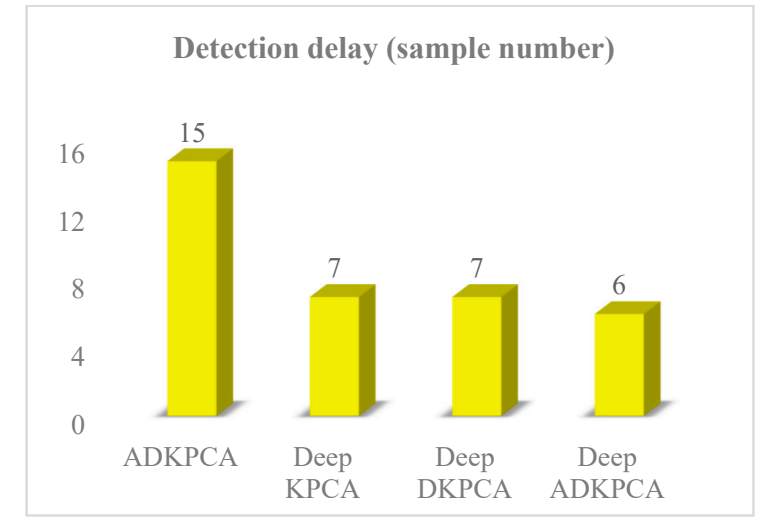

(b)

Figure 8. (a)The fault detection rate of rolling bearing outer ring fault and (b) the detection delay of rolling bearing outer ring fault.

Figure $8 \mathrm{~b}$ shows the detection delay of the rolling bearing outer ring fault by ADKPCA, Deep KPCA, Deep DKPCA, and Deep ADKPCA method. The ADKPCA method detected a failure at the fifth augmented data point, that is, at approximately the 15th sampling point. We could obtain that the Deep KPCA and Deep DKPCA methods were the 7th sampling points. The Deep ADKPCA method detected a failure at the second augmented data point, that is, at approximately the 6th sampling point. It can be seen from the failure delay that the Deep ADKPCA method could detect the incipient failure of the rolling bearing outer ring earlier.

Therefore, the validity and accuracy of proposed methods were better than that of other methods.

\subsection{Simulation Results and Analysis of The Rolling Bearing Rolling Body Faults}

\subsubsection{The Rolling Body Fault Simulation Results}

The fault characteristics of rolling elements were more complicated, which was determined by the motion characteristics of rolling elements. When the rolling bearing is in operation, it must not only ensure the rotation with the cage, but also complete its own rotation [36]. When a rolling body fails, the modulation frequency of its high-frequency resonance signal will be consistent with the rotation frequency of the cage, and the signal in the frequency domain will have a larger amplitude spectral line. Theoretically, the sideband distance on both sides of the spectral line should be consistent with the rotation frequency of the cage. However, due to the interference of the rolling motion of the rolling body, the performance of the actual collected vibration signal had weak characteristics. Fault detection was difficult. This section tested the incipient failure of rolling elements to verify the accuracy of the proposed Deep ADKPCA method. The simulation results were as follows.

The validity and accuracy of proposed methods were tested, and the results were also compared with other known methods such as ADKPCA, Deep KPCA, and Deep DKPCA.

Figure 9 describes the simulation results of the Deep ADKPCA fault detection method for the rolling bearing rolling body. Figure $9 \mathrm{a}, \mathrm{b}$ shows the simulation results of $T_{01}^{2}$ and $Q_{01}$ statistics of the original space $X$ based on the Deep ADKPCA method, that is, the simulation results based on the ADKPCA method. Figure $9 \mathrm{c}-\mathrm{j}$ shows the simulation results of $T_{i j}^{2}$ and $Q_{i j}$ statistics of the second-order subspace $X_{21}-X_{24}$ based on the Deep ADKPCA method, respectively. It can be seen from Figure $9 \mathrm{a}, \mathrm{b}$ that the failure detection rate of the ADKPCA method was about $50 \%$, this method basically did not have incipient fault detection capabilities. Figure $9 \mathrm{c}$ shows the detection result of the fault by the $T_{21}^{2}$ statistic. It can be seen from the image that the $T_{21}^{2}$ statistic was less sensitive to the fault than other 
statistic. Figure $9 \mathrm{~g}, \mathrm{i}, \mathrm{h}$ shows $T_{23}^{2}, T_{24}^{2}$, and $Q_{23}$ statistics for the fault detection result. From the images we could obtain that $T_{21}^{2}, T_{24}^{2}$, and $Q_{23}$ were more sensitive to this fault, and the fault detection rate was about $85 \%$. So, the Deep ADKPCA method had a good incipient fault detection capability.

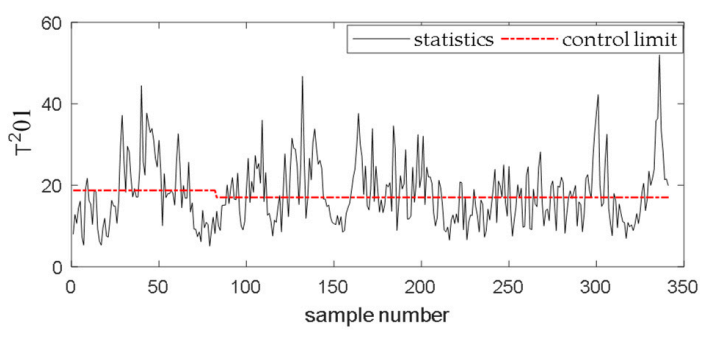

(a)

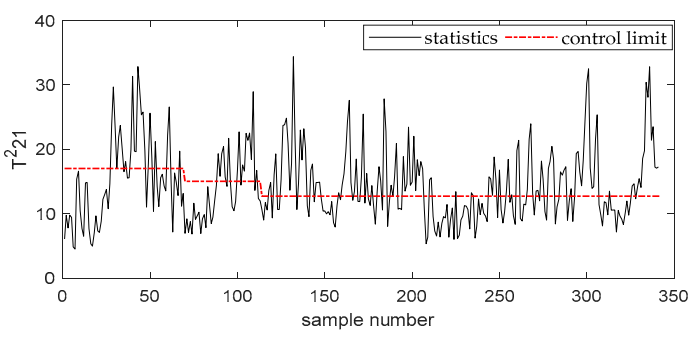

(c)

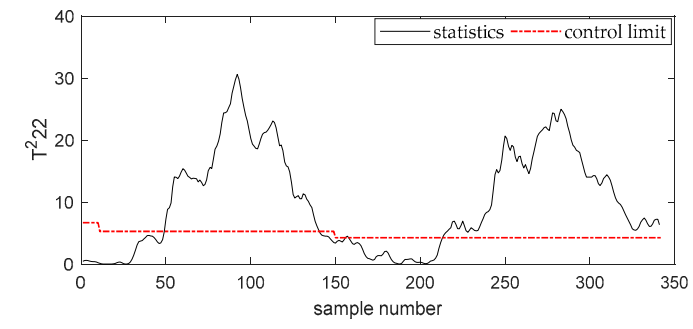

(e)

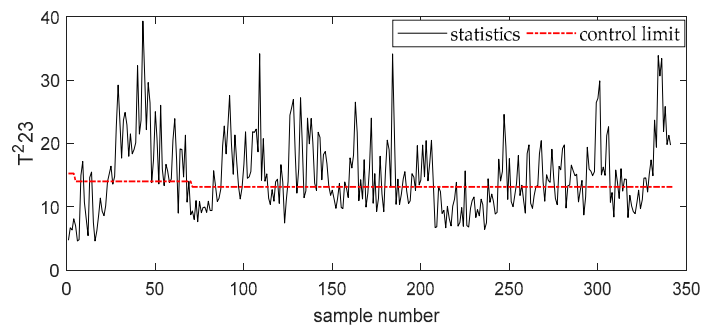

(g)

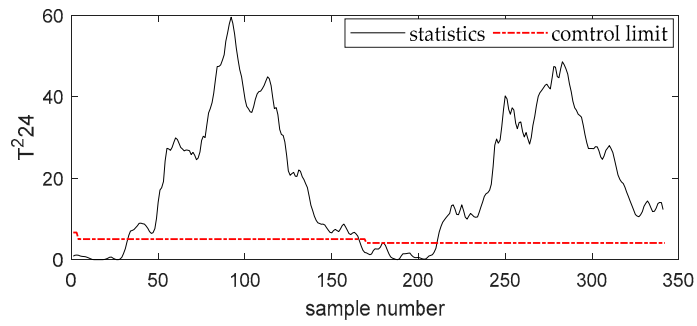

(i)

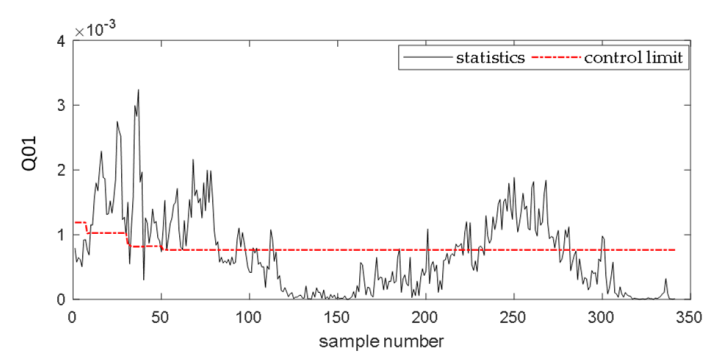

(b)

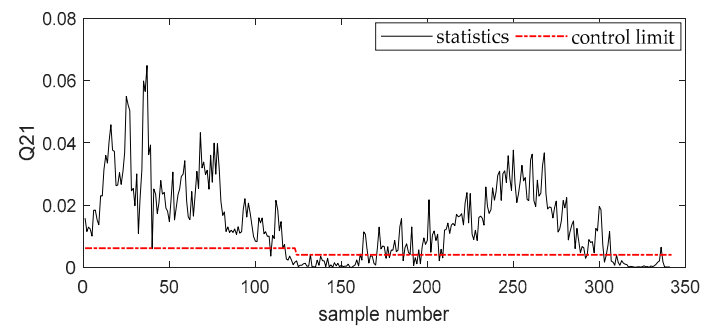

(d)

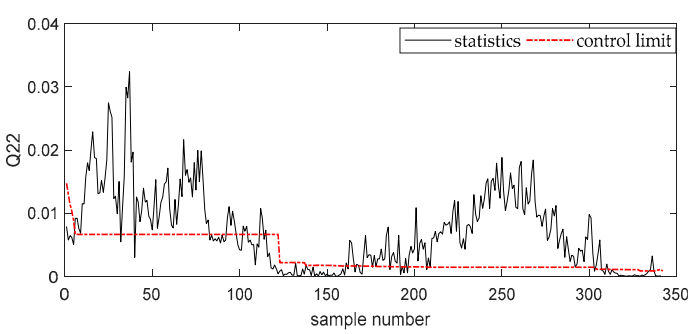

(f)

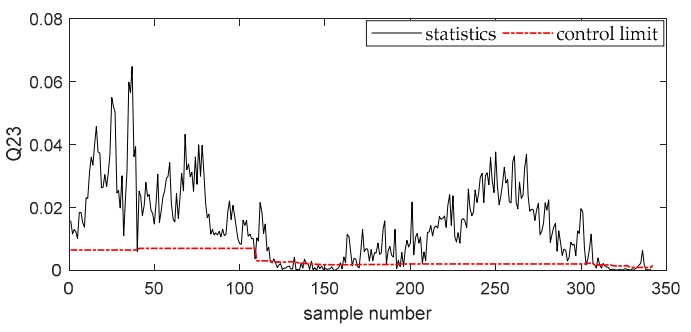

(h)

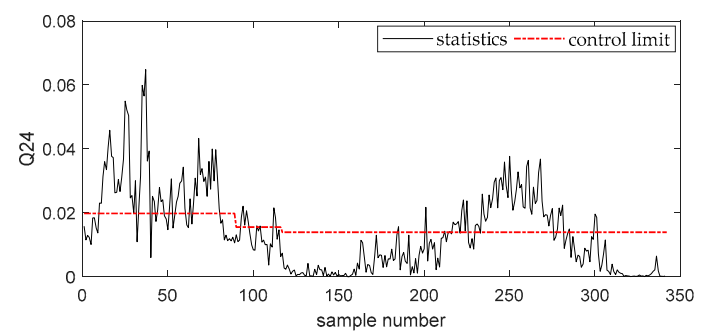

(j)

Figure 9. Experimental results of the Deep ADKPCA fault detection method for the rolling bearing rolling body fault. (a) The result of $T_{01}^{2}$, (b) The result of $Q_{01}$, (c) The result of $T_{21^{\prime}}^{2}$ (d) The result of $Q_{21}$, (e) The result of $T_{22^{\prime}}^{2}$ (f) The result of $Q_{22}$, (g) The result of $T_{23^{\prime}}^{2}$ (h) The result of $Q_{23}$, (i) The result of $T_{24}^{2}$ and (j) The result of $Q_{24}$. 
Figure 10 describes the simulation results of the Deep KPCA fault detection method for the rolling bearing rolling body. Figure $10 \mathrm{a}-\mathrm{h}$ describes the simulation results of $T_{i j}^{2}$ and $Q_{i j}$ statistics of the second-order subspace $X_{21}-X_{24}$ based on the Deep KPCA method, respectively. Figure 10c,e shows the detection results of incipient failures of rolling bearing outer rings of $T_{22}^{2}$ and $T_{23}^{2}$, we could obtain that $T_{22}^{2}$ and $T_{23}^{2}$ were less affected by this fault. From Figure $10 \mathrm{~g}$, we could obtain that $T_{24}^{2}$ was more sensitive to the fault. The Deep KPCA method had some incipient fault detection capabilities, but it still had some disadvantages compared to the Deep ADKPCA method.

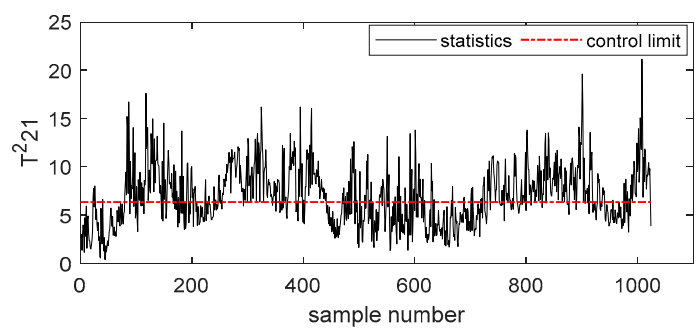

(a)

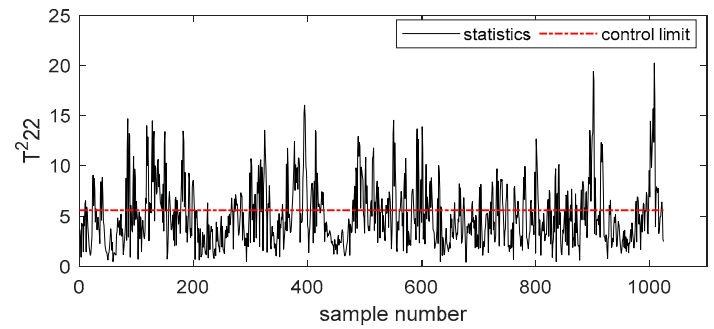

(c)

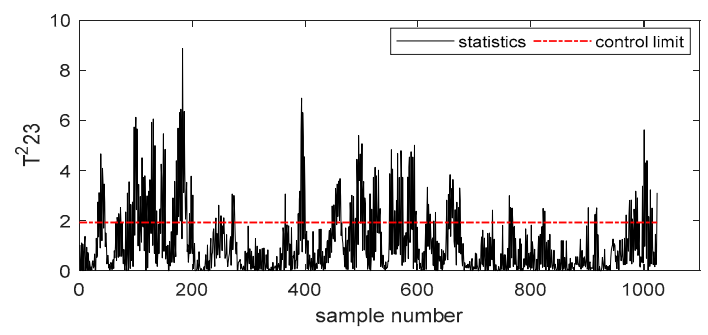

(e)

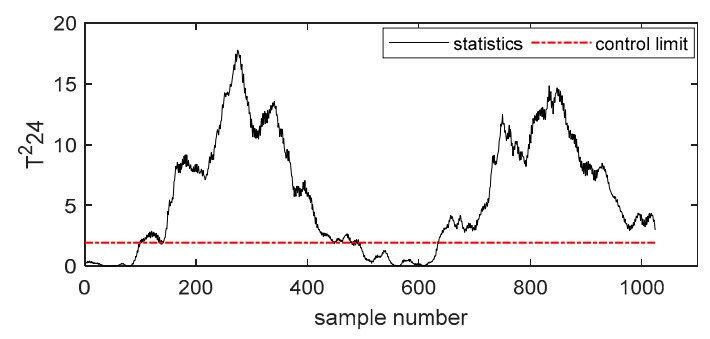

(g)

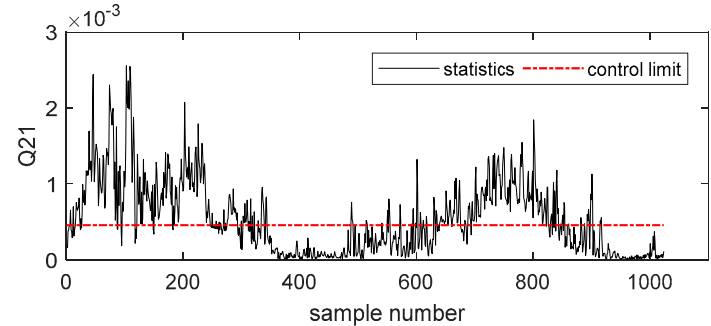

(b)

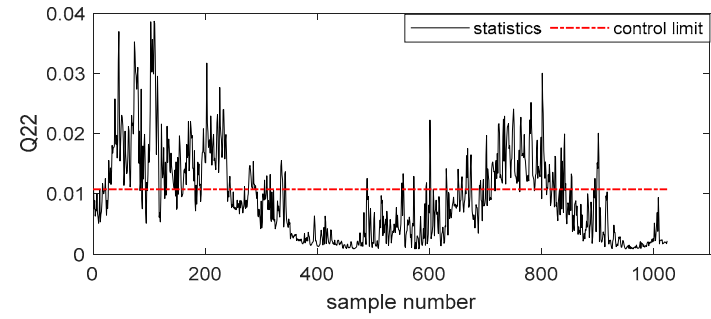

(d)

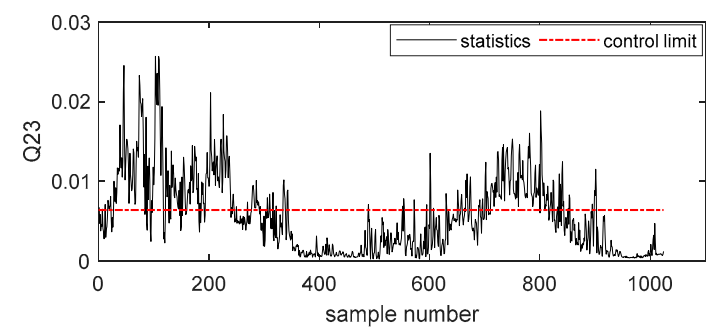

(f)

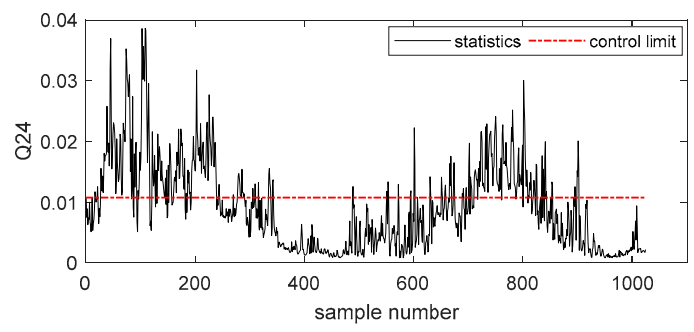

(h)

Figure 10. Experimental results of the Deep KPCA fault detection method for the rolling bearing rolling body fault. (a) The result of $T_{21^{\prime}}^{2}$ (b) The result of $Q_{21}$, (c)The result of $T_{22^{\prime}}^{2}$ (d) The result of $Q_{22}$, (e) The result of $T_{23^{\prime}}^{2}$ (f) The result of $Q_{23}$, (g) The result of $T_{24}^{2}$ and (h) The result of $Q_{24}$.

Figure 11 describes the simulation results of the Deep DKPCA fault detection method for the rolling bearing rolling body. Figure $11 \mathrm{a}-\mathrm{h}$ describes the simulation results of $T_{i j}^{2}$ and $Q_{i j}$ statistics of the second-order subspace $X_{21}-X_{24}$ based on the Deep DKPCA method, respectively. Figure 11b,g describes the detection results of incipient failures of rolling bearing outer rings of $Q_{21}$ and $T_{24^{\prime}}^{2}$ the rolling bearing outer rings fault could be successfully detected by $Q_{21}$ and $T_{24}^{2}$. From Figure $11 \mathrm{e}$, 
we could obtain that this fault had no obvious influence on $T_{23}^{2}$. Compared with the Deep KPCA method, the incipient fault detection capability of Deep DKPCA method was improved. This is because the Deep DKPCA method considered the timing correlation characteristic, but it still had some disadvantages compared to the Deep ADKPCA method.

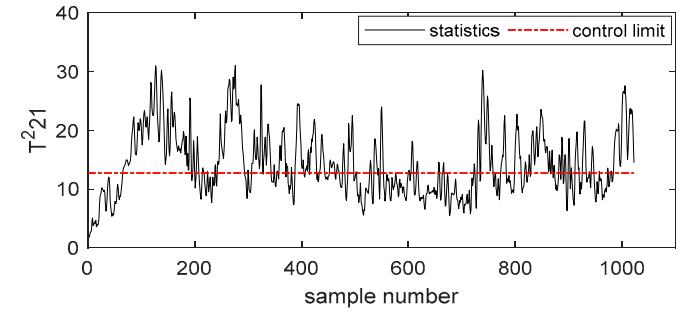

(a)

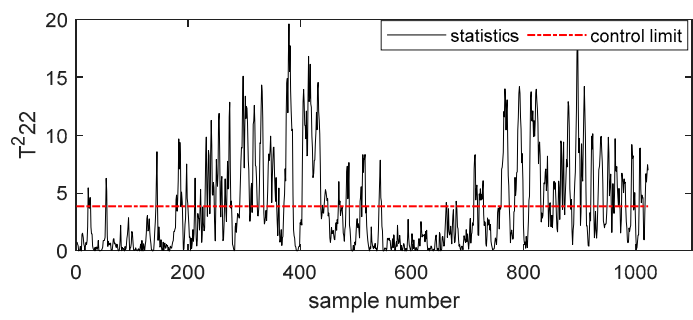

(c)

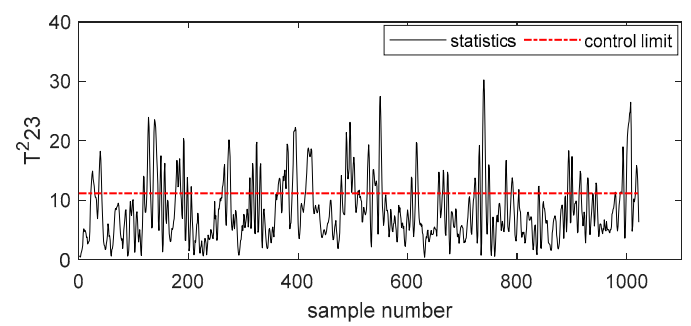

(e)

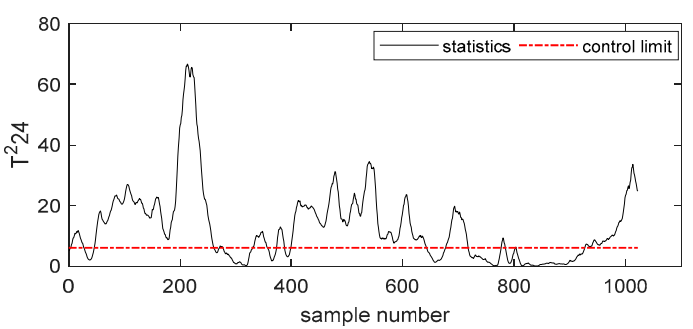

(g)

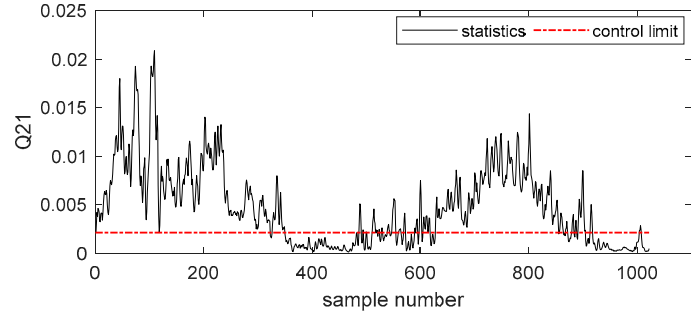

(b)

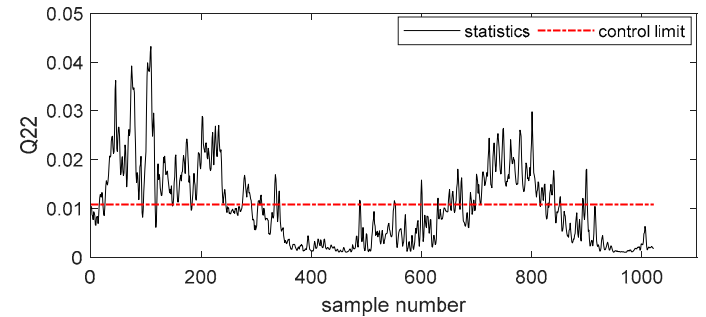

(d)

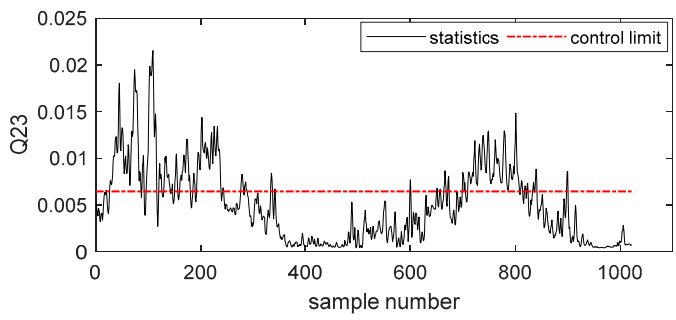

(f)

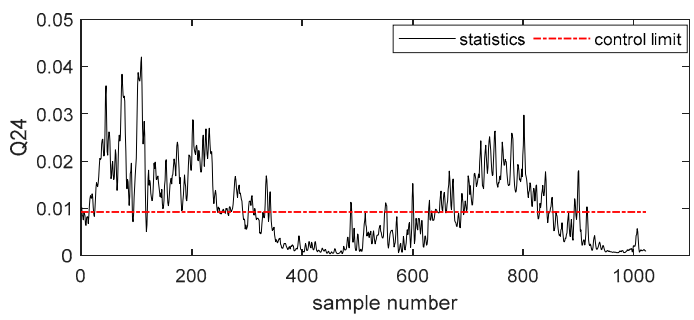

(h)

Figure 11. Experimental results of the Deep DKPCA fault detection method for the rolling bearing rolling body fault. (a) The result of $T_{21^{\prime}}^{2}$ (b) The result of $Q_{21}$, (c) The result of $T_{22^{\prime}}^{2}$ (d) The result of $Q_{22}$,

(e) The result of $T_{23^{\prime}}^{2}$ (f) The result of $Q_{23}$, (g) The result of $T_{24}^{2}$ and (h) The result of $Q_{24}$.

\subsubsection{Performance Comparison}

This section compared the experimental results of ADKPCA, Deep KPCA, Deep DKPCA, and Deep ADKPCA by analyzing the failure detection rate and detection delay of the incipient failure experimental results of rolling bearing rolling elements.

Figure 12a shows the fault detection rate of the rolling bearing rolling body fault by ADKPCA, Deep KPCA, Deep DKPCA, and Deep ADKPCA method. The failure detection rate of the ADKPCA failure detection method was $57.14 \%$, which proved that this method basically did not have incipient fault detection capability. The fault detection rate of Deep KPCA and Deep DKPCA was about 80\%, compared with ADKPCA method the detection rate had increased, but the detection capability still 
needs to be improved. Deep ADKPCA had a fault detection rate of $85.71 \%$, which was significantly higher than other methods, and had a certain incipient fault detection capability.

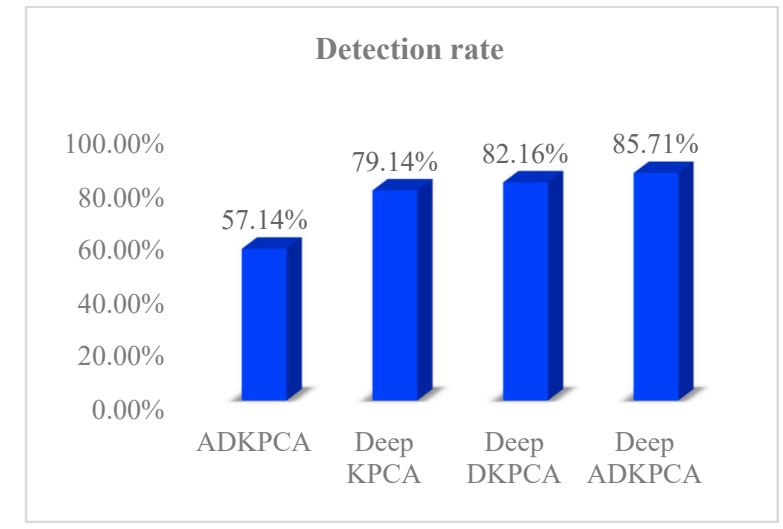

(a)

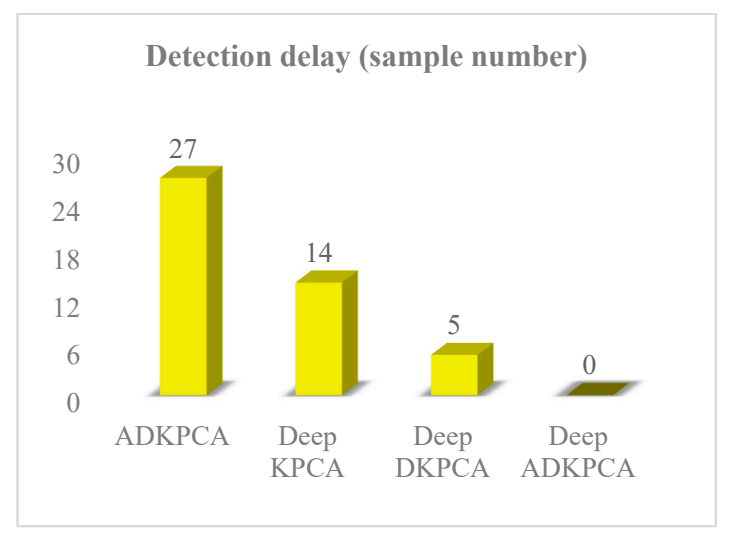

(b)

Figure 12. (a) The fault detection rate of the rolling bearing rolling body fault and (b) the detection delay of the rolling bearing outer rolling body fault.

Figure $12 \mathrm{~b}$ shows the detection delay of the rolling bearing rolling body fault by ADKPCA, Deep KPCA, Deep DKPCA, and Deep ADKPCA method. The ADKPCA method detected a failure at the 9th augmented data point, that is, at about the 27th sampling point. The Deep KPCA and Deep DKPCA methods found failures at the 14th and 5th sampling points, respectively. These methods could quickly detect the incipient fault. The Deep ADKPCA method detected failures at the beginning of the inspection without a time delay. From Figure $9 \mathrm{~b}$ we could obtain that $Q_{21}$ was more sensitive to this fault, the fault was detected from the beginning of the fault without delay. It could be seen from the failure delay that the Deep ADKPCA method could detect the incipient failure of the rolling bearing outer ring earlier.

It can be seen that Deep ADKPCA method could quickly detect the fault than the other three methods, and its detection rate was also the higher among the four methods. In summary, Deep ADKPCA had good fault detection performance.

\section{Conclusions}

Aiming at the problems that the incipient fault characteristics are weak, and the vibration signals of rolling bearings are a set of time series with non-linear and timing correlation, a nonlinear dynamic incipient fault detection method based on Deep ADKPCA was proposed. This method combines the advantages of Deep PCA and moving window algorithm, effectively captures the noise information by capturing the timing relationship between variables, fully mines the fault information in the signal, and improves the incipient detection capability of the fault. The proposed method uses the concept of kernel function, constructs a dynamic non-linear principal component model, and has the ability to handle non-linear and non-stationary signals. Compared with the fault detection results of the ADKPCA, Deep KPCA, and Deep DKPCA methods, the fault detection rate of the Deep ADKPCA method proposed in this paper was more than $85 \%$, the detection delay was almost zero, and the ability to detect incipient faults was significantly improved. It was proved that Deep ADKPCA incipient fault detection method had a certain feasibility. The application of this method in other fields such as motors and rotors needs further research.

Author Contributions: Conceptualization, H.S. and X.B.; software, J.G.; validation, J.G., X.B., and L.G.; formal analysis, H.S., X.B., and Z.L.; resources, H.S., X.B., and J.S.; data curation, L.G.; writing-original draft preparation, J.G.; writing-review and editing, H.S. and X.B.; supervision, H.S. and X.B. All authors have read and agreed to the published version of the manuscript. 
Acknowledgments: This research was funded by National Science Foundation of China (Nos: 51705341, 51905357, 51675353), Natural Science Foundation of Liaoning Province (No: 2019-ZD-0654).

Conflicts of Interest: The authors declare no conflict of interest

\section{References}

1. Islam, R.; Kim, Y.-H.; Kim, J.; Kim, J.-M. Detecting and Learning Unknown Fault States by Automatically Finding the Optimal Number of Clusters for Online Bearing Fault Diagnosis. Appl. Sci. 2019, 9, 2326. [CrossRef]

2. Li, Y.; Yang, Y.; Wang, X.; Liu, B.; Liang, X. Early fault diagnosis of rolling bearings based on hierarchical symbol dynamic entropy and binary tree support vector machine. J. Sound Vib. 2018, 428, 72-86. [CrossRef]

3. Wang, Y.; Tang, B.; Qin, Y.; Huang, T. Rolling Bearing Fault Detection of Civil Aircraft Engine Based on Adaptive Estimation of Instantaneous Angular Speed. IEEE Trans. Ind. Inform. 2020, 16, 4938-4948. [CrossRef]

4. Li, H.; Liu, T.; Wu, X.; Chen, Q. Research on bearing fault feature extraction based on singular value decomposition and optimized frequency band entropy. Mech. Syst. Signal Process. 2019, 118, 477-502. [CrossRef]

5. Mao, W.; Chen, J.; Liang, X.; Zhang, X. A New Online Detection Approach for Rolling Bearing Incipient Fault via Self-Adaptive Deep Feature Matching. IEEE Trans. Instrum. Meas. 2020, 69, 443-456. [CrossRef]

6. Tang, L.; Chen, A.Q.; Li, D.J. Time-varying tan-type barrier Lyapunov function-based adaptive fuzzy control for switched systems with unknown dead zone. IEEE Access 2019, 7, 110928-110935. [CrossRef]

7. Chen, Y.; Zhang, T.; Luo, Z.; Sun, K. A novel rolling bearing fault diagnosis and severity analysis method. Appl. Sci. 2019, 9, 2356. [CrossRef]

8. Duan, Z.; Wu, T.; Guo, S.; Shao, T.; Malekian, R.; Li, Z. Development and trend of condition monitoring and fault diagnosis of multi-sensors information fusion for rolling bearings: A review. Int. J. Adv. Manuf. Technol. 2018, 96, 803-819. [CrossRef]

9. Shi, H.; Bai, X.; Zhang, K.; Wu, Y.; Yue, G. Influence of uneven loading condition on the sound radiation of starved lubricated full ceramic ball bearings. J. Sound Vib. 2019, 461, 114910. [CrossRef]

10. Li, H.; Niu, Y.; Li, Z.; Xu, Z.; Han, Q. Modeling of amplitude-dependent damping characteristics of fiber reinforced composite thin plate. Appl. Math. Model. 2020, 80, 394-407. [CrossRef]

11. Shi, H.; Bai, X. Model-based uneven loading condition monitoring of full ceramic ball bearings in starved lubrication. Mech. Syst. Signal Process. 2020, 139, 106583. [CrossRef]

12. Tan, D.; Li, L.; Yin, Z.; Li, D.; Zhu, Y.; Zheng, S. Ekman boundary layer mass transfer mechanism of free sink vortex. Int. J. Heat Mass Transf. 2020, 150, 119250. [CrossRef]

13. Skóra, M.; Ewert, P.; Kowalski, C.T. Selected Rolling Bearing Fault Diagnostic Methods in Wheel Embedded Permanent Magnet Brushless Direct Current Motors. Energies 2019, 12, 4212. [CrossRef]

14. Wang, X.; Qin, Y.; Wang, Y.; Xiang, S.; Chen, H. ReLTanh: An activation function with vanishing gradient resistance for SAE-based DNNs and its application to rotating machinery fault diagnosis. Neurocomputing 2019, 363, 88-98. [CrossRef]

15. Shi, H.; Guo, L.; Tan, S.; Bai, X.; Sun, J. Rolling Bearing Initial Fault Detection Using Long Short-Term Memory Recurrent Network. IEEE Access 2019, 7, 171559-171569. [CrossRef]

16. Zhou, Q.; Shen, H.; Zhao, J.; Liu, X.; Xiong, X. Degradation State Recognition of Rolling Bearing Based on K-Means and CNN Algorithm. Shock. Vib. 2019, 2019, 8471732. [CrossRef]

17. Eren, L.; Ince, T.; Kiranyaz, S. A Generic Intelligent Bearing Fault Diagnosis System Using Compact Adaptive 1D CNN Classifier. J. Signal Process. Syst. 2018, 91, 179-189. [CrossRef]

18. Zheng, K.; Luo, J.; Zhang, Y.; Li, T.; Wen, J.; Xiao, H. Incipient fault detection of rolling bearing using maximum autocorrelation impulse harmonic to noise deconvolution and parameter optimized fast EEMD. ISA Trans. 2019, 89, 256-271. [CrossRef]

19. Wang, Y.; Tse, P.W.; Tang, B.; Qin, Y.; Deng, L.; Huang, T. Kurtogram manifold learning and its application to rolling bearing weak signal detection. Measurement 2018, 127, 533-545. [CrossRef]

20. Cui, L.; Wang, X.; Wang, H.; Ma, J. Research on Remaining Useful Life Prediction of Rolling Element Bearings Based on Time-Varying Kalman Filter. IEEE Trans. Instrum. Meas. 2019. [CrossRef] 
21. Wang, D.; Tsui, K.-L. Dynamic Bayesian wavelet transform: New methodology for extraction of repetitive transients. Mech. Syst. Signal Process. 2017, 88, 137-144. [CrossRef]

22. Li, G.; Tang, G.; Luo, G.; Wang, H. Underdetermined blind separation of bearing faults in hyperplane space with variational mode decomposition. Mech. Syst. Signal Process. 2019, 120, 83-97. [CrossRef]

23. Li, Y.; Xu, M.; Wei, Y.; Huang, W. Health Condition Monitoring and Early Fault Diagnosis of Bearings Using SDF and Intrinsic Characteristic-Scale Decomposition. IEEE Trans. Instrum. Meas. 2016, 65, 2174-2189. [CrossRef]

24. Chen, F.; Cheng, M.; Tang, B.; Xiao, W.; Chen, B.; Shi, X. A novel optimized multi-kernel relevance vector machine with selected sensitive features and its application in early fault diagnosis for rolling bearings. Meas. 2020, 156, 107583. [CrossRef]

25. Yu, K.; Lin, T.R.; Ma, H.; Li, H.; Zeng, J. A Combined Polynomial Chirplet Transform and Synchroextracting Technique for Analyzing Nonstationary Signals of Rotating Machinery. IEEE Trans. Instrum. Meas. 2020, 69, 1505-1518. [CrossRef]

26. Chen, H.; Jiang, B.; Lu, N.; Jiang, B. Deep PCA Based Real-Time Incipient Fault Detection and Diagnosis Methodology for Electrical Drive in High-Speed Trains. IEEE Trans. Veh. Technol. 2018, 67, 4819-4830. [CrossRef]

27. Liu, L.; Liu, Y.; Li, D.; Tong, S.; Wang, Z. Barrier Lyapunov Function-Based Adaptive Fuzzy FTC for Switched Systems and Its Applications to Resistance-Inductance-Capacitance Circuit System. IEEE Trans. Cybern. 2019, 1-12. [CrossRef]

28. Liu, Y.; Zhao, Y.; Li, J.; Ma, H.; Yang, Q.; Yan, X. Application of weighted contribution rate of nonlinear output frequency response functions to rotor rub-impact. Mech. Syst. Signal Process. 2020, 136, 106518. [CrossRef]

29. Tang, L.; Ma, D.; Zhao, J. Adaptive neural control for switched non-linear systems with multiple tracking error constraints. IET Signal Process. 2019, 13, 330-337. [CrossRef]

30. Zheng, J.D.; Pan, H.Y.; Qi, X.L.; Zhang, X.Q.; Liu, Q.Y. Enhanced empirical wavelet transform based time-frequency analysis and its application to rolling bearing fault diagnosis. Tien Tzu Hsueh Pao/acta Electron. Sin. 2018, 46, 358-364.

31. Schölkopf, B.; Smola, A.; Müller, K.-R. Nonlinear Component Analysis as a Kernel Eigenvalue Problem. Neural Comput. 1998, 10, 1299-1319. [CrossRef]

32. Xie, L.; Tao, J.; Zhang, Q.; Zhou, H. CNN and KPCA-Based Automated Feature Extraction for Real Time Driving Pattern Recognition. IEEE Access 2019, 7, 123765-123775. [CrossRef]

33. Tang, L.; Li, D. Time-varying Barrier Lyapunov Function Based Adaptive Neural Controller Design for Nonlinear Pure-feedback Systems with Unknown Hysteresis. Int. J. Control. Autom. Syst. 2019, 17, 1642-1654. [CrossRef]

34. Song, K.; Xu, P.; Chen, Y.; Zhang, T.; Wei, G.; Wang, Q. A Fault Diagnosis and Reconfiguration Strategy for Self-Validating Hydrogen Sensor Array Based on MWPCA and ELM. IEEE Access 2019, 7, 115075-115092. [CrossRef]

35. El Laithy, M.; Wang, L.; Harvey, T.J.; Vierneusel, B.; Correns, M.; Blass, T. Further understanding of rolling contact fatigue in rolling element bearings-A review. Tribol. Int. 2019, 140, 105849. [CrossRef]

36. Yu, K.; Ma, H.; Zeng, J.; Han, H.; Li, H.; Wen, B. Frobenius and nuclear hybrid norm penalized robust principal component analysis for transient impulsive feature detection of rolling bearings. ISA Trans. 2019. [CrossRef]

(C) 2020 by the authors. Licensee MDPI, Basel, Switzerland. This article is an open access article distributed under the terms and conditions of the Creative Commons Attribution (CC BY) license (http://creativecommons.org/licenses/by/4.0/). 